\title{
A deficiência de vitamina A em crianças no Brasil eno mundo
}

\author{
Vitamin A deficiency among children in Brazil and worldwide
}

Regina Célia Rodrigues M iranda M ilagres ${ }^{1}$

Lélia Cápua Nunes ${ }^{2}$

Helena Maria Pinheiro-Sant'Ana ${ }^{1}$

${ }^{1}$ Departamento de

Abstract Based on a ten-year literature review, this paper describes important aspects of Vitamin A D eficiency (VAD) among children in Brazil and worldwide. It presents VAD within a clinical and sub-clinical deficiency context, emphasizing this as a public health issue. VAD has been diagnosed in children from several parts of Brazil, especially in the Southeast and Northeast. Worldwide, the highest VAD prevalence is found in parts of parts of Africa: M ali, Ethiopia and Nigeria, and clinical indications noted especially in parts of Asia and Africa. However, no studies of clinical indications were located. Studies point to VAD as a public health issue among children, particularly in the poorest parts of the world.

Key words Vitamin A deficiency, Children, Clinical indications, Sub-clinical indications, Public health issues
Resumo 0 presente artigo expõe, com base em uma revisão de literatura dos últimos dez anos, aspectos relevantes da Deficiência de Vitamina A (DVA) em crianças no Brasil eno mundo. Apresenta a DVA no contexto da deficiência clínica e subclínica, com ênfase na caracterização desta como problema de saúde pública. A DVA foi diagnosticada em crianças de várias regiões do Brasil, principalmente no Sudeste e N ordeste; entretanto, não foram encontrados estudos relativos a sinais clínicos. M undialmente, as mai ores prevalências de DVA foram registradas em países da África como Mali, Etiópia e Nigéria, sendo que os sinais clínicos estiveram presentes, principalmente, em regiões da Ásia e África. Os estudos apontam a DVA como problema de saúde pública em crianças, principalmente nas regiões mais pobres do globo.

Palavras-chave Deficiência de vitamina A, Crianças, Sinais clínicos, Sinais subclínicos, Problema de saúde pública 


\section{Introdução}

A deficiência de vitamina A (DVA) é considerada um problema grave em mais de sessenta países ${ }^{1}$. Deacordo com a Organização Mundial de Saúde, cerca de 2,8 milhões de crianças em idade préescolar no mundo são clinicamente afetadas pela hipovitaminoseA. Estima-se que de 250 a 500 mil crianças tornem-se cegas todos os anos, sendo que cerca da metade morrem antes de completar um ano de vida?

Esta deficiência pode ser causada por dois fatores principais. 0 primeiro por umaingestão inadequada de vitamina A para satisfazer as necessidades orgânicas, como o consumo insuficiente de produtos de origem animal e de frutas e hortaliças ricas em pró-vitamina $A$, levando a uma ineficiente absorção deste micronutriente. 0 segundo está relacionado ao sinergismo entre episódios infecciosos e a carência de vitamina A (adaptado de Ramalho e Saunders ${ }^{3}$ ).

Em crianças em idade préescolar, este distúrbio nutricional pode causar aumento do risco de mortalidade, morbidadee cegueira. As conseqüências para crianças em idade escolar e adolescentes têm sido menos estudadas e são provavelmente menos intensas4.

Diante da magnitude desta deficiência, percebe-se a importância de conhecer os indicadores do estado nutricional de vitamina A que podem ser utilizados no diagnóstico desta deficiência. Deacordo com a U NICEF 5 , o uso dos indicadores como sinais clínicos (xeroftalmia, manchas de Bitot e cegueira noturna) e avaliação bioquímica fornecida pelos níveis de retinol no sangue podem determinar a DVA como problema de saúde pública. Os indicadores citológicos (impressão conjuntival) e os dietéticos (inquéritos qua- litativosequantitativos) também têm sido comumente empregados para detectar a carência de vitamina $A^{6}$.

A classificação da xeroftalmia e as prevalências mínimas para definir a deficiência de vitamina A como importante problema de saúde pública em crianças menores de seis anos, de acordo com a World Health Organization ${ }^{7}$ (WH O), estão descritas na Tabela 1.

Em relação aos níveis séricos de retinol, a $\mathrm{WHO}^{7}$ sugere os seguintes critérios (Tabela 2 ), sendo que a prevalência de níveis séricos de retinol $<0,70 \mu \mathrm{mol} / \mathrm{L}$ em $2 \%$ a $10 \%$ da população infantil de 6 a 71 meses de idade indica problema de saúde pública leve, de $10 \%$ a 20\%, problema moderado e $>20 \%$, grave?

Ainda que inquéritos nacionais sejam escassos, no Brasil, a prevalência de DVA está estimada entre 16 e $74 \%$ em crianças menores de seis anos ${ }^{8}$. De acordo com a Organização Pan-Americana de Saúde(OPAS) ea Organização Mundial de Saúde (OM S), o Brasil é considerado como área de carência subclínica grave 9 .

Diante desses dados, o presente estudo traça um perfil de informações relacionadas com a distribuição da DVA em crianças, de acordo com dados clínicos e subclínicos no Brasil e no mundo. Assim, foi realizado um levantamento bibliográfico de artigos encontrados nas bases de dados Medline, Lilacs, PubM ed, Scielo e Science Direct e publicações de organizações governamentais, que abordam dados de prevalência da DVA em crianças, totalizando 100 referências pesquisadas. Deste total, 70 foram selecionados por apresentarem maior ligação com o tema estudado. Foi feita uma seleção deartigos publicados nosúltimos dez anos, de forma a priorizar dados mais recentes e atualizados sobre o tema.

Tabela 1. Classificação da xeroftalmia e prevalência mínima em crianças menores de seis anos para definir a deficiência de vitamina A como problema de saúde pública.

\begin{tabular}{lrr}
\hline Classificação & Indicador & Prevalência mínima \\
\hline XN & Cegueira noturna & $1 \%$ \\
X1A & Xerose conjuntival & Não usado \\
X1B & M ancha de Bitot & $0,5 \%$ \\
X2 & Xerose corneal & $0,01 \%$ \\
X3A & Ulceração corneal/queratomalácea & $0,01 \%$ \\
X3B & $<1 / 3$ da superfície corneal & \\
XS & Ulceração corneal/queratomalácea & $0,01 \%$ \\
XF & $\geq 1 / 3$ da superfície corneal & \\
& Cicatriz corneal & $0,05 \%$ \\
\hline
\end{tabular}

Fonte: Organização Mundial da Saúde (1996)? 
Tabela 2. Classificação de níveis séricos de retinol.

\begin{tabular}{lrr}
\hline \multirow{2}{*}{ Classificação } & \multicolumn{2}{c}{ Nível de Retinol Sérico } \\
\cline { 2 - 3 } & \multicolumn{1}{c}{$\mu \mathrm{g} / \mathrm{dL}$} & \multicolumn{1}{c}{$\mu \mathrm{mol} / \mathrm{L}$} \\
\hline Normal & $>30,0$ & $>1,05$ \\
Aceitável & 20,0 a 29,9 & 0,70 a 1,04 \\
Baixo & 10,0 a 19,9 & 0,35 a 0,69 \\
Deficiente & $<10,0$ & ou $<0,35$
\end{tabular}

Fonte: Organização Mundial da Saúde (1996) ${ }^{7}$.

\section{A deficiência de vitamina A no Brasil}

Os dados dos últimos vinte anos indicam que a DVA éum problema de saúde pública no Brasil ${ }^{10}$, principalmentenas regiões Norte, Nordeste e Sudeste, sendo possível identificar a população infantil do Nordeste como a mais vulnerável ao problema, uma vez que entre $16 \%$ a $55 \%$ das crianças apresentaram níveis séricos de retinol abaixo de $20 \mu \mathrm{g} / \mathrm{dL}$, o que caracteriza uma carência endê mica $^{11}$. De acordo com Ramalho et al. ${ }^{8}$, os inqué ritos bioquímicos disponíveis no Brasil confirmam que a DVA é um problema de saúde pública nos estados de São Paulo, M inas Gerais, Pernambuco, Paraíba, Bahia e Amazonas.

Gonçalves-Carvalho et al. ${ }^{12}$ estudaram 131 crianças de 3 a 10 anos, da periferia do município de Campinas, São Paulo. A prevalência de hipovitaminose A foi observada em $17,6 \%$ das crianças, com níveis deretinol sérico entre 0,35 e $0,70 \mu \mathrm{mol} /$ L, distribuídosem 6,9\% para préescolarese $10,7 \%$ para escolares. Outro estudo de prevalência realizado em crianças usuárias da rede básica de saúde de sete municípios de São Paulo com idades entre 6 e 23 meses mostrou que $30 \%$ das crianças apresentaram níveis séricos de retinol abaixo de 0,70 $\mu \mathrm{mol} / \mathrm{L}$ e $7 \%$ abaixo de $0,35 \mu \mathrm{mol} / \mathrm{L}^{13}$.

Em Ribeirão Preto (SP), níveis de retinol sérico considerados baixos foram detectados em $21,4 \%$ das 103 crianças de 6 a 24 meses estudadas. A freqüência foi maior entre crianças que nasceram com baixo peso e que tiveram aleitamento materno por um menor período de tempo ${ }^{14}$. Em uma avaliação prospectiva de 47 pacientes com déficit estatural de um ambulatório da capital paulista, crianças com idade variando entre 4 e 14 anos foram avaliadas quanto ao nível sérico de retinol. A freqüência de níveis de retinol $<1,05$ $\mu \mathrm{mol} / \mathrm{L}$ foi encontrada em $21 \%$ dos pacientes, mas não houverelação dosníveis séricos deretinol com a velocidade de crescimento ${ }^{15}$. Vítolo et al. ${ }^{16}$ investigaram os níveis séricos de retinol de 218 crianças eadolescentes com idades entre $10 \mathrm{e} 19$ anos, matriculados em escola particular também da capital paulista. Os resultados mostraram que 10\% dos investigados apresentaram valores abaixo de $0,70 \mu \mathrm{mol} / \mathrm{L}$ e $30 \%$ abaixo de $1,05 \mu \mathrm{mol} / \mathrm{L}$.

No que se refere ao estado de M inas Gerais, apenas o estudo de Santos et al. ${ }^{17}$ foi encontrado neste período. Os resultados revelaram que $29 \%$ dos 241 escolares de 6 a 14 anos, da zona rural de Novo Cruzeiro, tinham níveis de retinol abaixo de $20 \mu \mathrm{g} / \mathrm{dL}$.

A DVA também foi pesquisada por Ramalho et al. ${ }^{18}$, em um estudo realizado em 253 recémnascidos assistidos em duas maternidades públicas do município do Rio de Janeiro. Os autores constataram que a maioria $(55,7 \%)$ dos recémnascidos apresentou valores de retinol sérico abaixo de $1,05 \mu \mathrm{mol} / \mathrm{L}$, sendo que $1,23 \%$ tiveram valores abaixo de 0,35 $\mu \mathrm{mol} / \mathrm{L}$. Os recém-nascidos com baixo peso ao nascer apresentaram tendência a uma maior prevalência de hipovitaminose $A$ $(68,7 \%)$ em comparação àqueles com peso adequado (54\%).

Ainda no município do Rio de Janeiro, 175 préescolares atendidos em uma Unidade de Saúde foram avaliados quanto à prevalência de DVA, revelando níveis séricos $<1,05 \mu \mathrm{mol} / \mathrm{L}$ em 34,3\% das crianças atendidas ${ }^{19}$. Outro estudo, realizado no Rio de Janeiro por Ramalho et al. ${ }^{20} \mathrm{em} 574$ escolares de 7 a 17 anos de uma rede municipal de ensino, constatou níveis baixos de retinol sérico em $10,28 \%$ das crianças, sendo que houve uma tendência a maiores percentuais de inadequação sérica de retinol entre escolares mais jovens ( $11,98 \%$ na faixa etária de 7 a 10 anos e 7,92\% na faixa etária de 10 a 17 anos).

Consideração importante deve ser direcionada aos estados do Nordeste. De acordo com Ferraz et al. ${ }^{14}$, a DVA, principalmente a forma subclínica, éendêmica em váriasáreas do Nordeste brasileiro. $\mathrm{Na}$ Bahia, uma pesquisa realizada na cidade de Cansanção, com 61 crianças de 6 a 72 meses de idade, demonstrou a ocorrência de níveis inadequados de retinol sérico (deficientes $<10 \mu \mathrm{g} /$ $\mathrm{dL}$ e baixos $<20 \mu \mathrm{g} / \mathrm{dL}$ ) em $44,7 \%$ das crianças, principal mente entre crianças menores de $24 \mathrm{me}$ ses, caracterizando a deficiência como problema de saúde pública ${ }^{21}$.

Uma pesquisa ampla envolvendo sete municípios do semi-árido baiano contou com a participação de 563 préescolares. Os resultados revelaram que a prevalência de níveis deficientes de retinol sérico $(<10 \mu \mathrm{g} / \mathrm{dL})$ foi de $15,3 \%$, sendo 
que a maior prevalência foi detectada em Valente $(31,9 \%$ ) e a menor em Serrinha (7,8\%). Em relação à faixa etária, a maior prevalência deníveis de retinol sérico deficientes foi registrada na faixa de 24 a 35 meses (22,9\%) e a menor na faixa de 48 a 59 meses $(7,8 \%)^{22}$. Assis et al. ${ }^{23}$ pesquisaram crianças de 6 a 72 meses dessa mesma região da Bahia e encontraram prevalência de $40,4 \%$ de crianças com níveis séricos de retinol considerados baixos (0,35 a 0,69 $\mu \mathrm{mol} / \mathrm{L})$ e 4,3\% com níveis deficientes $(<0,35 \mu \mathrm{mol} / \mathrm{L})$.

Em vinte conglomerados do estado de Sergipe, M artins et al. ${ }^{24}$ demonstraram quedos 607 pré escolares de 6 a 60 meses, $22,5 \%$ das crianças tiveram níveis séricos de retinol considerados baixos, enquanto que os níveis considerados deficientes foram detectados em 9,6\% dos casos. No total, a prevalência DVA foi de $32,1 \%$ em todas as crianças pesquisadas. A prevalência deníveis inadequados de vitaminaA detectada nessa população classifica a DVA como um importante problema de saúde pública.

Diniz $^{25}$, ao estudar crianças menores de cinco anos na Paraíba, encontrou prevalência de $16,0 \%$ de DVA. Essa faixa etária também foi avaliada no estado de Pernambuco em um estudo realizado pelo INAN/M S-IM IP ${ }^{26}$, que encontrou $19,3 \%$ das crianças com níveis séricos de retinol abaixo de 0,70 $\mu \mathrm{mol} / \mathrm{L}$ e $3 \%$ com níveis abaixo de $0,35 \mu \mathrm{mol} / \mathrm{L}$, constituindo, portanto, um problema de saúde pública.

No estado de Pernambuco, 311 crianças menores de cinco anos de creches públicas da cidade de Recife foram pesquisadas quanto a DVA em relação aos indicadores bioquímicos (retinol sérico), dietéticos (inquérito de consumo alimentar) e antropométricos (peso/altura, altura/idade e peso/altura). Os resultados revelaram a prevalência de níveis séricos de retinol baixos em $7 \%$ das crianças, caracterizando a DVA como problema de saúde pública leve $e^{27}$.

0 diagnóstico do estado nutricional no estado do Amazonas em relação a hipovitaminose $A$ foi realizado por Alencar et al. ${ }^{28}$. Os autores fizeram uma investigação clínico-oftalmológica envolvendo 487 crianças de 2 a 5 anos de idade e a dosagem de retinol sérico de 48 crianças do município de Nhamundá. A investigação clínica não evidenciou sinal eou sintoma pertinentesà carência de vitaminaA. Níveis deretinol sérico considerados altos ( $>50,0 \mu \mathrm{g} / \mathrm{dL}$ ) foram detectados em $25 \%$ das crianças e níveis considerados aceitáveis (20,0 a 29,9 $\mu \mathrm{g} / \mathrm{dL})$, em $75 \%$ delas. Assim, não se pode afirmar a existência de hipovitaminose $A$ como problema de saúde pública nesta região.
Apesar de não terem caráter nacional, dados levantados neste estudo sugerem quea DVA é um problema de saúde pública em várias regiões do Brasil. A Tabela 3 descreve o resumo dos inquéritos pesquisados sobre sua prevalência no Brasil.

\section{Programas de cor reção da DVA} por órgãos governamentais no Brasil

Como forma de atenção à correção da DVA no Brasil, destacam-se programas como o de Combate à Deficiência de Vitamina $A$, instituído pela Portaria do M inistério da Saúde, n² 2.160 de 29 de dezembro de $1994{ }^{29}$. Recentemente, foi instituído o Programa Nacional de Suplementação de Vitamina A, através da Portaria do M inistério da Saúde ${ }^{\circ} 729$, de 13 demaio de 2005. Esse programa destina-se a prevenir e/ou controlar essa deficiência nutricional mediante a suplementação com megadoses de vitamina $A$, em crianças de 6 a 59 meses de idade e puérperas no pós-parto imediato, pertencentes à Região Nordeste, ao Vale do Jequitinhonha, M inas Gerais eao Vale do Ribeira, São Paulo ${ }^{30}$.

\section{A deficiência de vitamina A no mundo}

A DVA é uma das mais importantes e prevalentes deficiências e tem sido considerada um problema de saúde pública entre crianças pré-escolares, em países em desenvolvimento ${ }^{31,32}$. Estimativas recentes indicam que mundialmente mais de dois milhões de pessoas possuem risco de desenvolver DVA e deficiência de iodo ou ferro, apesar de recentes esforços na prevenção e controle dessas deficiências. A prevalência é especialmentealta no Sudeste da Ásia e na região do Saara, na Áfri$\mathrm{ca}^{32,33}$. Gestantes e crianças jovens representam o maior grupo de risco 33,35 .

Dados levantados por West J r. ${ }^{33}$ revelaram maiores prevalências de crianças pré-escolares com DVA na Etiópia (61,2\%), no Quênia (40,6\%) ena Indonésia ( $57 \%$ ). Outra análise demonstrou quea prevalência deDVA entre pré-escolares, utilizando ponto de corte de $<0,70 \mu \mathrm{mol} / \mathrm{L}$, foi maior em Gana (73,3\%), Papua N ova Guiné $(60,2 \%)$, Sul da África (48,2\%), Nepal $(37,2 \%)$ e Paquistão $(31,8 \%)^{36}$.

Poucos são os dados disponíveis sobre a prevalência da DVA entre crianças em todas as regiões do mundo ${ }^{37}$; entretanto, o presente trabalho procurou identificar estudos da ocorrência desta doença em alguns continentes. 
Tabela 3. Deficiência de vitamina A no Brasil.

\begin{tabular}{|c|c|c|c|c|c|}
\hline Estado & Faixa etária & Amostra & $\begin{array}{l}\text { Prevalência } \\
\text { de DVA }\end{array}$ & $\begin{array}{l}\text { Indicadores/ } \\
\text { ponto de corte }\end{array}$ & Ano e Fonte \\
\hline \multicolumn{6}{|l|}{ São Paulo } \\
\hline $\begin{array}{l}\text { Campinas } \\
\text { (Periferia) }\end{array}$ & 3-10 anos & 131 & $17,6 \%$ & $\mathrm{RS}^{*}$ 0,35 a 0,70 $\mu \mathrm{mol} / \mathrm{L}$ & $\begin{array}{r}\text { Gonçalves-Carvalho } \\
\text { et al., } 1995\end{array}$ \\
\hline $\begin{array}{l}\text { Ribeirão Preto } \\
\text { (Clínica infantil) }\end{array}$ & 6-24 meses & 103 & $21,4 \%$ & $\mathrm{RS}^{*}<0,70 \mu \mathrm{mol} / \mathrm{L}$ & Ferraz et al., 2000 \\
\hline $\begin{array}{l}\text { São Paulo } \\
\text { (Escola particular) }\end{array}$ & 10-19 anos & 218 & $\begin{array}{l}10 \% \\
30 \%\end{array}$ & $\begin{array}{l}\mathrm{RS}^{*}<0,70 \mu \mathrm{mol} / \mathrm{L} \\
\mathrm{RS}^{*}<1,05 \mu \mathrm{mol} / \mathrm{L}\end{array}$ & Vítolo et al., 2004 \\
\hline $\begin{array}{l}\text { São Paulo } \\
\text { ( } 7 \text { municípios) }\end{array}$ & 6-23 meses & & $\begin{array}{r}30 \% \\
7 \%\end{array}$ & $\begin{array}{l}\mathrm{RS}^{*}<0,70 \mu \mathrm{mol} / \mathrm{L} \\
\text { RS* }^{*}<0,35 \mu \mathrm{mol} / \mathrm{L}\end{array}$ & Souza et al., 1998 \\
\hline $\begin{array}{l}\text { São Paulo } \\
\text { (Ambulatório) }\end{array}$ & 4-14 anos & 47 & $21 \%$ & $\mathrm{RS}^{*}<1,05 \mu \mathrm{mol} / \mathrm{L}$ & Sarni et al., 2002 \\
\hline \multicolumn{6}{|l|}{ Minas Gerais } \\
\hline $\begin{array}{l}\text { Novo Cruzeiro } \\
\text { (Zona rural) }\end{array}$ & $6-14$ anos & 241 & $29 \%$ & $\mathrm{RS}^{*}<20 \mu \mathrm{g} / \mathrm{dL}$ & Santos et al., 2005 \\
\hline \multicolumn{6}{|l|}{ Bahia } \\
\hline $\begin{array}{l}\text { Cansanção } \\
\text { (Áreas rurais) }\end{array}$ & $6-72$ meses & 61 & $44,7 \%$ & $\mathrm{RS}^{*}<20 \mu \mathrm{g} / \mathrm{dL}$ & Prado et al., 1995 \\
\hline $\begin{array}{l}7 \text { M unicípios do semi-árido } \\
\text { (área urbana) }\end{array}$ & Préescolares & 563 & $15,3 \%$ & $\mathrm{RS}^{*}<10 \mu \mathrm{g} / \mathrm{dL}$ & Santos et al., 1996 \\
\hline $\begin{array}{l}7 \text { M unicípios do semi-árido } \\
\text { (área urbana) }\end{array}$ & $6-72$ meses & & $\begin{array}{r}40,4 \% \\
4,3 \%\end{array}$ & $\begin{array}{r}\text { RS* 0,35 a 0,69 } \mu \mathrm{mol} / \mathrm{L} \\
\mathrm{RS}^{*}<0,35 \mu \mathrm{mol} / \mathrm{L}\end{array}$ & Assis et al., 1997 \\
\hline $\begin{array}{l}\text { Sergipe } \\
\text { (16 municípios) }\end{array}$ & 6 - 60 meses & 607 & $\begin{array}{r}22,5 \% \\
9,6 \%\end{array}$ & 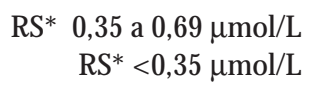 & M Martins et al., 2004 \\
\hline $\begin{array}{l}\text { Recife } \\
\text { (Creches públicas) }\end{array}$ & $<5$ anos & 311 & $7,0 \%$ & $\mathrm{RS}^{*}<0,70 \mu \mathrm{mol} / \mathrm{L}$ & $\begin{array}{r}\text { Fernandes et } \\
\text { al.,2005 }\end{array}$ \\
\hline Pernambuco & $<5$ anos & & $\begin{array}{r}19,3 \% \\
3 \%\end{array}$ & $\begin{array}{l}\mathrm{RS}^{*}<0,70 \mu \mathrm{mol} / \mathrm{L} \\
\mathrm{RS}^{*}<0,35 \mu \mathrm{mol} / \mathrm{L}\end{array}$ & $\begin{array}{r}\text { INAN/M S-IM IP, } \\
1998\end{array}$ \\
\hline \multicolumn{6}{|l|}{ Rio de Janeiro } \\
\hline $\begin{array}{l}\text { Rio de Janeiro } \\
\text { ( } 2 \text { maternidades) }\end{array}$ & Neonatais & 253 & $\begin{array}{l}55,7 \% \\
1,23 \%\end{array}$ & $\begin{array}{l}\mathrm{RS}^{*}<1,05 \mu \mathrm{mol} / \mathrm{L} \\
\mathrm{RS}^{*}<0,35 \mu \mathrm{mol} / \mathrm{L}\end{array}$ & Ramalho et al., 1998 \\
\hline $\begin{array}{l}\text { Rio de Janeiro } \\
\text { (Unidade de Saúde) }\end{array}$ & Préescolares & 175 & $34,3 \%$ & $\mathrm{RS}^{*}<1,05 \mu \mathrm{mol} / \mathrm{L}$ & Ramalho et al., 2001 \\
\hline $\begin{array}{l}\text { Rio de Janeiro } \\
\text { (Rede M unicipal de Ensino) }\end{array}$ & $7-17$ anos & 574 & $10,28 \%$ & $\mathrm{RS}^{*}<1,05 \mu \mathrm{mol} / \mathrm{L}$ & Ramalho et al., 2004 \\
\hline $\begin{array}{l}\text { Amazonas } \\
\text { N hamundá }\end{array}$ & $2-5$ anos & 48 & $\begin{array}{l}25 \% \\
75 \%\end{array}$ & 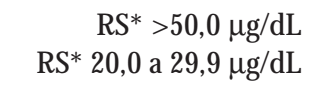 & Alencar et al., 2002 \\
\hline
\end{tabular}

*RS - Retinol Sérico. 
América

A DVA na América Latina e no Caribe foi estudada por Mora et al. ${ }^{38}$. Os resultados da pesquisa revelaram que a DVA nessa região é predominantemente subclínica e que a prevalência nacional de níveis de retinol sérico $<20 \mu \mathrm{g} / \mathrm{dL}$ em crianças menores de cinco anos oscila de 6,1\% no Panamá a 36\% em El Salvador. 0 problema é grave em cinco países, moderado em seis e leve em quatro. A população afetada é de aproximadamente 14,5 milhões de crianças menores decinco anos (25\%). De acordo com esses autores, as medidas para controlar a DVA incluem suplementação, fortificação do açúcar e algumas atividades de diversificação alimentar.

$\mathrm{NaC}$ olômbia, DeNavarro et al. ${ }^{39}$ pesquisaram a deficiência de ferro, vitamina $A$ e prevalência de parasitismo intestinal, constatando $13,2 \%$ de níveis séricos de retinol sérico $<70 \mu \mathrm{mol} / \mathrm{L}$ entre 2.187 pré-escolares. Os níveis de vitamina A, C e folato em uma amostra de 1966 crianças até 12 anos, no M éxico, foram avaliados por Villalpando et al ${ }^{40}$. Os resultados revelaram que níveis de retinol sérico $<10 \mu \mathrm{g} / \mathrm{dL}$ foram raros entre as crianças pesquisadas; entretanto, a deficiência subclínica (retinol entre $>10 \mu \mathrm{g} / \mathrm{dL}$ e $<20 \mu \mathrm{g} / \mathrm{dL}$ ) esteve presenteem $25 \%$ das crianças de 1 a 8 anos. De acordo com os autores, a alta prevalência de DVA indica problema de saúde pública.

Estudo realizado com 590 crianças menores de 15 anos em Valência, na Venezuela, demonstrou que $0,7 \%$ tinham níveis baixos de retinol sérico ( $<20 \mu \mathrm{g} / \mathrm{dL}$ ) e 25,1\% tinham níveis aceitáveis de retinol (entre 20 e $30 \mu \mathrm{g} / \mathrm{dL}$ ) ${ }^{41}$. Os resultados dos sinais clínicos de impressão citológica conjuntival revelaram que $11,1 \%$ das crianças estavam deficientes. Ainda na Venezuela, Castejon et al. ${ }^{42}$ pesquisaram a coexistência de anemia, DVA e retardo no crescimento entre 202 crianças de 24 a 84 meses em Maracaibo, demonstrando a ocorrência de 21,78\% de DVA (retinol sérico < $20 \mu \mathrm{g}$ / $\mathrm{dL}$ ), 38,11\% deanemia e $14,36 \%$ de déficit no crescimento. Em outro estudo realizado em M aracaibo, em 157 crianças de 2 a 6 anos das zonas rural e urbana, não foram evidenciados sinais clínicosou oftalmológicos de DVA. A prevalência de DVA subclínica foi de $35,4 \%$, sendo maior em crianças do meio rural (48,3\%). Esses dadosindicam quea DVA é um problema de saúde pública em algumas regiões da Venezuela. Assim, a implementação de um programa nutricional integral que inclua suplementação, fortificação de alimentos, diversificação dietética, melhora das condições so- cioeconômicas esanitárias etambém do nível educacional, com ênfase na educação em saúde e nutrição, é recomendado por esses autores ${ }^{43}$.

Stephens et al. ${ }^{44}$ chamaram a atenção para a subestimação do número de casos de carência subclínica de vitamina $A$ inclusive em países desenvolvidos, como os Estados Unidos, onde a ingestão inadequada de retinol e carotenóides é o principal fator etiológico da DVA.

Ásia

A DVA existe como problema nutricional de saúde pública entre crianças em idade pré-escolar em 118 países em desenvolvimento, como a região do Sudeste da Ásia (Bangladesh, Butão, Índia, Indonésia, República Democrática Popular da Coréia, Maldivas, União do M iamar, Nepal, Sri Lanka e Tailândia), que abrange o maior número de $\operatorname{casos}^{32}$.

A deficiência subclínica de vitamina A (retinol sérico $<20 \mu \mathrm{g} / \mathrm{dL}$ ) tem sido registrada mundialmente como um aumento no risco de morbidades e mortalidades em crianças ${ }^{45}$. Entretanto, o impacto da DVA durante a gravidez na saúde fetal não tem sido documentado ${ }^{31}$. Estudos realizados por Stephenson et al. ${ }^{45}$ revelaram que níveis de retinol sérico $<20 \mathrm{mg} / \mathrm{dL}$ em mulheres podem estar associados com aumento do risco de transferência para o feto e anemia na mãe. Feungpean et al..$^{46}$ demonstraram que $16 \%$ de 19 crianças prematuras de Bangcoc, na Tailândia, tinham níveis plasmáticos de vitamina $\mathrm{A}$ abaixo de $20 \mathrm{mg} / \mathrm{dL}$ após setedias denascidas eque, após catorze etrinta dias, todos os prematuros tinham níveis normais. Resultados semelhantes foram evidenciados em Israel, onde a deficiência subclínica de vitamina $A$ (retinol sérico $<0,7 \mathrm{mmol}$ / L) foi encontrada em mais de $15 \%$ das 117 das crianças nômades, entre 0 e 18 meses estudadas, utilizando esse mesmo ponto de corte ${ }^{47}$.

Uma revisão de literatura sobre a ocorrência deDVA e a xeroftalmia no sudeste da Ásia foi realizada por Singh $\&$ West J r. ${ }^{37}$. A prevalência estimada de DVA entre crianças em idade escolar foi de $23,4 \%$ ea de xeroftalmia branda (cegueira noturna ou mancha de Bitot) foi de $2,6 \%$, concluindo quea DVA entre escolares pode ser considerada um importante problema de saúde pública no sudeste da Ásia.

No caso particular da Índia, estudos têm evidenciado a DVA como um problema importante em crianças. Em uma tribo da Índia, crianças em idade pré-escolar foram pesquisadas quanto ao 
estado nutricional. U m total de 1.022 crianças de 27 vilas foi pesquisado ea DVA em forma de manchas de Bitot foi encontrada em 1,6\% das crianças $^{48}$. De acordo com Singh \& West J r. ${ }^{37}$, a prevalência de xeroftalmia entre crianças em idade escolar naĺndia é de 2,8\%.

Um estudo multicêntrico, que avaliou a prevalência de outros sinais clínicos presentes na DVA, como mancha de Bitot e cicatriz corneal (em 164.512 crianças menores de 6 anos) e cegueira noturna (entre 113.202 crianças de 24 a 71 meses), foi conduzido durante os anos de 1997 a 2000, em crianças de dezesseis regiões de onze estados da Índia. A maior prevalência foi constatada em Gaya, onde 4,71\% das crianças apresentaram mancha de Bitot, 0,5\% cicatriz corneal e $5,17 \%$ cegueira noturna ${ }^{49}$.

Sinais clínicos também foram estudados por Bhattacharya et al..$^{50} \mathrm{em} 331$ estudantes primários de uma região de Naxalbari em Bengal Ocidental, na Índia. Os resultados revelaram que $3,65 \%$ das crianças tinham acuidade visual anormal; 8,16\% apresentaram DVA e 3,63\% estavam com mancha de Bitot. Em pesquisa baseada em impressão citológica conjuntival realizada em Nagpur na Índia, foi demonstrado que a prevalência deDVA foi estimada em 35,7\% de 308 crianças de área urbana, abaixo de 6 anos ${ }^{51}$. Pesquisa envolvendo sinais clínicos da DVA entre 700 crianças iraquianas menores de 6 anos revelou que a xeroftalmia foi prevalente em $29 \%$ das crianças, sendo maior entre 1 e 3 anos $^{52}$.

$\mathrm{Na}$ China, a deficiência subclínica de vitamina A foi pesquisada por Tan et al. ${ }^{53}$. Os autores identificaram em uma amostra de 8.669 crianças abaixo de 6 anos de catorze províncias chinesas a prevalência de $11,7 \%$ de DVA utilizando o ponto de corte de $\leq 0,70 \mu \mathrm{mol} / \mathrm{L}$. A prevalênciafoi maior em crianças do meio rural (15\%) em comparação com crianças do meio urbano (5,2\%). Khatib ${ }^{54}$, ao pesquisar um grupo de 1.023 crianças de 5,5 a 9,9 anos quanto à deficiência subclínica de vitamina A em sete regiões semi-rurais da Jordânia, encontrou prevalência superior $(21,8 \%)$.

A idade e a estação do ano parecem influenciar na prevalência de DVA em crianças. No Paquistão, 154 crianças de 0 a 15 anos que apresentavam DVA foram pesquisadas. Os resultados demonstraram que crianças abaixo de 6 anos são mais vulneráveis e que a ocorrência da deficiência é maior no verão $(39 \%)^{55}$.

A DVA foi considerada por $M$ ihora et al. ${ }^{56}$ como um moderado problema de saúde pública em crianças enutrizes na cidade deCabul, no Afe- ganistão. Algumas medidas vêm sendo bastante positivas na diminuição da prevalência de DVA, tais como programas de suplementação de vitamina A. Dados da Filipinas demonstraram quea prevalência de DVA em crianças de 1 a 5 anos estava em torno de $38 \%$ (níveis de retinol sérico $<20 \mu \mathrm{g} / \mathrm{dL}$ ) em 1998. Este percentual foi reduzido para $32 \%$ após um a quatro meses de suplementação com vitamina $A^{56}$. Deacordo com $K$ han et al. ${ }^{58}$, a DVA é uma das maiores deficiências no Vietnã e um programa de suplementação de vitamina A pode ser implementado com sucesso na prevenção da DVA.

\section{África}

Estimativas da W $\mathrm{HO}^{2}$ revelaram que a DVA é considerada um problema de saúde pública em 44 países da África. 0 problema é particularmente grave no Mali ${ }^{59}$. Estudos conduzidos com 1.510 pré-escolares nesse país mostraram que $43,8 \%$ das crianças tinham níveis séricos de retinol abaixo de 0,35 $\mu \mathrm{mol} / \mathrm{L}$ e $92,7 \%$ tinham níveis abaixo de $0,70 \mu \mathrm{mol} / \mathrm{L}$. Em relação aos sinais clínicos, 4,3\% das crianças apresentaram cegueira noturna e $\%$ tinham manchas de Bitot. A xeroftalmia foi prevalente em $77,1 \%$ das crianças pesquisadas. Esses dados sugerem que a DVA é um grave problema de saúde pública na área de Bandiagra, no M ali60.

Poucas pesquisas sobre a DVA têm sido conduzidasna Nigéria ${ }^{61}$. Estudos realizados nesse país por estes autores, em 213 crianças entre seis meses e seis anos, revelaram que $26,8 \%$ tinham níveis séricos de retinol deficientes $(<10 \mu \mathrm{g} / \mathrm{dL}$ ), enquanto 47,9\% tinham níveis entre 10 e $19 \mu \mathrm{g} /$ $\mathrm{dL}$. Os autores sugerem que medidas de combate à DVA sejam tomadas, incluindo suplementação de vitamina $A$, em curto prazo, e educação nutricional ressaltando a importância do consumo defontes de pró-vitaminaA evitaminaA pré formada em alimentos, em longo prazo. U ma revisão sobre a importância da xeroftalmia como causa de cegueira em crianças, portanto, como um significante problema de saúde pública na Nigéria, foi realizada por Rabiu \& Kyari62, revelando que a xeroftalmia causada pela DVA éum problema predominante entre as crianças, e que 34 a $69 \%$ da cegueira infantil na Nigéria écausada pela opacidade corneal.

Em M oçambique, estima-se que 2,3 milhões de crianças abaixo de 5 anos tenham DVA. $\mathrm{Na}$ ausência de políticas apropriadas e programas de ação, a DVA pode ser atribuída como causa de mais de 30.000 mortes anualmente entre crian- 
ças menores de 5 anos, o que representa 34,8\% de todas as causas de mortalidade neste grupo de idade. A suplementação de vitamina A tem sido adotada como estratégia a médio e curto prazo para controle da DVA em crianças ${ }^{63}$.

Para determinar a prevalência de DVA entre crianças de 6 a 9 anos no norte da Etiópia ${ }^{64}$, foram pesquisadas 824 das 1.339 crianças para as quais existia informação completa dos níveis bioquímicos de vitamina A, exame ocular para xeroftalmia e dados antropométricos. A prevalência de xeroftalmia foi de 5,8\%. Níveis séricos de retinol abaixo de $0,35 \mu \mathrm{mol} / \mathrm{L}$ foram encontrados em $8,4 \%$ das crianças e níveis entre 0,35 e 0,70 $\mu \mathrm{mol} / \mathrm{L}$ foram encontrados em $51,1 \%$ das crianças.

Estudo com crianças em idade pré-escolar e escolar em Arsi na Etiópia concluiu que a DVA é um problema de saúde nessa região, pois foram encontradas prevalências de $7,2 \%$ decegueira noturna; $2,2 \%$ de mancha de Bitot; $0,2 \%$ de xerose corneal; $0,5 \%$ de ulceração corneal e 0,5\% de cicatriz corneal ${ }^{65}$. A alta prevalência de DVA entre crianças de 6 a 9 anos remeteà necessidade deprogramas de suplementação de vitamina $\mathrm{A}$ em áreas onde a DVA é endêmica. Isso é confirmado por Kello \& Gilbert ${ }^{66}$, que encontraram em um estudo com 360 escolares da Etiópia a DVA e o sarampo como as maiores causas de diminuição da visão e cegueira nessas crianças.

\section{Europa}

Esse continente não é considerado como área de risco; por este motivo, trabalhos que abordam a DVA em criança em países da Europa são escassos. A pesar disso, Gregory Jr et al. ${ }^{67}$ encontraram em 1.003 pré-escolares do Reino Unido a prevalência de 8,1\% de níveis séricos de retinol $<0,70$ $\mu \mathrm{mol} / \mathrm{L}$, demonstrando que a DVA nesta região é considerada um problema de saúde pública leve.

\section{Oceania}

Na República das I lhas M arshall, a DVA, deficiência de ferro e anemia foram pesquisadas em 20\% (919) das crianças de dez pequenas ilhas, com idades entre 1 e 5 anos. Os resultados demonstra- ram que a proporção de crianças com DVA (retinol sérico $<0,70 \mu \mathrm{mol} / \mathrm{L}$ ) foi de $59,9 \%{ }^{68}$. Deacordo com informações da XXII Reunião do "International Vitamin A Consultative Group" (IVACG $)^{69}$, em Pohnpei, na M icronésia, existe um sério problema de DVA relacionado ao aumento do consumo de arroz e alimentos importados, em substituição aos alimentos tradicionalmente consumidos na região.

Com objetivo de facilitar a análise dos dados, os estudos acima foram compilados nas Tabelas 4 e 5, que descrevem os resultados de sete investigações da América, doze da Ásia, sete da África, uma da Europa e uma da O ceania.

\section{Programas de cor reção da DVA por órgãos governamentais no mundo}

Como medida de controle a curto prazo para a DVA, a UNICEF, em parceria com a WHO e outros órgãos internacionais, tem implementado programas de suplementação de vitamina A para crianças de 6 a 59 meses de idade e puérperas no pós- parto imediato. Antes da implantação do programa em um determinado país, é feita uma avaliação de indicadores de DVA para certificar se a área é de risco ${ }^{5}$.

Por muitos anos, diversos países foram relutantes em iniciar a suplementação de vitamina A devido a dúvidas sobre a segurança desta iniciativa. M as os estudos mostraram que os benefícios da suplementação da vitamina A compensam todos os efeitos colaterais, que são muito raros ${ }^{70}$. A Tanzânia, Nepal e Vietnã são exemplos de países onde os programas foram bem-sucedidos ${ }^{5}$.

A UNICEF adota outras estratégias a longo prazo, como apontar a diversificação e fortificação dos alimentos. Muitos paísesnaAmérica Central, como Guatemala e Honduras, tiveram grande sucesso com a fortificação do açúcar ${ }^{5}$. 0 IVACG também enfatiza a fortificação de alimentos, como um método de combatea DVA, assim como a educação nutricional, e estudos relativos ao aumento da biodisponinilidade da vitamina A em alimentos ${ }^{69}$. 
Tabela 4. Deficiência de vitamina A no mundo, segundo a avaliação bioquímica.

\begin{tabular}{|c|c|c|c|c|c|}
\hline País & Idade & Amostra & $\begin{array}{l}\text { Prevalência } \\
\text { de DVA }\end{array}$ & $\begin{array}{l}\text { Indicadores/ } \\
\text { ponto de corte }\end{array}$ & Ano e Fonte \\
\hline \multicolumn{6}{|l|}{ América } \\
\hline Panamá & $<5$ anos & & $6,1 \%$ & $\mathrm{RS}^{*}<20 \mu \mathrm{g} / \mathrm{dL}$ & Mora et al., 1998 \\
\hline El Salvador & $<5$ anos & & $36 \%$ & $\mathrm{RS}^{*}<20 \mu \mathrm{g} / \mathrm{dL}$ & M ora et al., 1998 \\
\hline M éxico & 1 a 8 anos & 1966 & $25 \%$ & $\mathrm{RS}^{*}>10 \mathrm{e}<20 \mu \mathrm{g} / \mathrm{dL}$ & Villalpando et al., 2003 \\
\hline Venezuela (Valência) & $<15$ anos & 590 & $\begin{array}{r}0,7 \% \\
25,1 \%\end{array}$ & 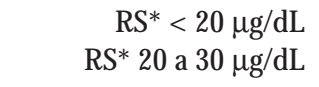 & Paez et al., 2002 \\
\hline Venezuela ( $M$ aracaibo) & 24 a 84 meses & 202 & $21,78 \%$ & $\mathrm{RS}^{*}<20 \mu \mathrm{g} / \mathrm{dL}$ & Castejon et al., 2004 \\
\hline Colômbia & Pré-escolares & 2187 & $13,2 \%$ & $\mathrm{RS}^{*}<0,70 \mu \mathrm{mol} / \mathrm{L}$ & De Navarro et al., 1996 \\
\hline \multicolumn{6}{|l|}{ Ásia } \\
\hline Filipinas & 1 a 5 anos & 117 & $38 \%$ & $\mathrm{RS}^{*}<20 \mu \mathrm{g} / \mathrm{dL}$ & Pedro et al., 2004 \\
\hline Israel (nômades) & 0 a 18 meses & 19 & $15,4 \%$ & $\mathrm{RS}^{*}<0,70 \mu \mathrm{mol} / \mathrm{L}$ & Coles et al., 2004 \\
\hline Tailândia & Prematuros & 8669 & $16 \%$ & $\mathrm{RS}^{*}<20 \mu \mathrm{g} / \mathrm{dL}$ & Feungpean et al., 2002 \\
\hline China (14 províncias) & $<$ Ganos & 1023 & $11,7 \%$ & $\mathrm{RS}^{*} \leq 0,70 \mu \mathrm{mol} / \mathrm{L}$ & Tan Z et al., 2002 \\
\hline Jordânia (7 regiões) & 5,5 a 9,9 anos & & $21,8 \%$ & & Khatib, 2002 \\
\hline Sudeste da Ásia & Escolares & & $23,4 \%$ & $\mathrm{RS}^{*}<0,70 \mu \mathrm{mol} / \mathrm{L}$ & Singh \& West Jr., 2004 \\
\hline \multicolumn{6}{|l|}{ África } \\
\hline Mali (Bandiagra) & Pré-escolares & 192 & $\begin{array}{l}43,8 \% \\
92,7 \%\end{array}$ & $\begin{array}{l}\mathrm{RS}^{*}<0,35 \mu \mathrm{mol} / \mathrm{L} \\
\mathrm{RS}^{*}<0,70 \mu \mathrm{mol} / \mathrm{L}\end{array}$ & Schémann et al., 2002 \\
\hline Etiópia (Arsi) & $\begin{array}{r}\text { Pré-escolares } \\
\text { e escolares }\end{array}$ & 402 & $51 \%$ & $\mathrm{RS}^{*}<20 \mu \mathrm{g} / \mathrm{dL}$ & Asrat et al., 2002 \\
\hline Nigéria (área rural) & 6 meses e 6 anos & 213 & $\begin{array}{l}26,8 \% \\
47,9 \%\end{array}$ & $\begin{array}{r}\text { RS }^{*}<10 \mu \mathrm{g} / \mathrm{dL} \\
\text { RS }^{*} 10 \text { a } 19 \mu \mathrm{g} / \mathrm{dL}\end{array}$ & Oso et al., 2003 \\
\hline Etiópia & 6 a 9 anos & 824 & $\begin{array}{r}8,4 \% \\
51,1 \%\end{array}$ & $\begin{array}{r}\mathrm{RS}<0,35 \mu \mathrm{mol} / \mathrm{L} \\
\text { RS* } 0,35 \text { a } 0,70 \mu \mathrm{mol} / \mathrm{L}\end{array}$ & Kassaye et al., 2001 \\
\hline \multicolumn{6}{|l|}{ Europa } \\
\hline Reino Unido & Pré-escolares & 1003 & $8,1 \%$ & $\mathrm{RS}^{*}<0,70 \mu \mathrm{mol} / \mathrm{L}$ & Gregory Jr et al., 1995 \\
\hline \multicolumn{6}{|l|}{ Oceania } \\
\hline I lhas M arshall & 1 a 5 anos & 919 & $59,9 \%$ & $\mathrm{RS}^{*}<0,70 \mu \mathrm{mol} / \mathrm{L}$ & Palafox et al., 2003 \\
\hline
\end{tabular}

*RS - Retinol Sérico. 
Tabela 5. Deficiência de vitamina A no mundo, segundo os sinais clínicos.

\begin{tabular}{|c|c|c|c|c|c|}
\hline País & Idade & Amostra & $\begin{array}{c}\text { Prevalência } \\
\text { de DVA }\end{array}$ & $\begin{array}{l}\text { Indicadores/ } \\
\text { ponto de corte }\end{array}$ & Ano e Fonte \\
\hline \multicolumn{6}{|l|}{ América } \\
\hline $\begin{array}{l}\text { Venezuela } \\
\text { (M aracaibo) }\end{array}$ & 2 a 6 anos & 157 & $35,4 \%$ & ICC* & Castejon et al., 2004 \\
\hline \multicolumn{6}{|l|}{ Ásia } \\
\hline \multirow{2}{*}{$\begin{array}{l}\text { Índia } \\
\text { (16 regiões } \\
\text { de } 11 \text { estados) }\end{array}$} & $<6$ anos & 164.512 & $\begin{array}{r}4,71 \% \\
0,5 \%\end{array}$ & $\begin{array}{l}\text { M anchas de Bitot } \\
\text { Cicatriz corneal }\end{array}$ & Toteja et al., 2002 \\
\hline & 24 a 71 meses & 113.202 & $5,17 \%$ & Cegueira noturna & \\
\hline $\begin{array}{l}\text { Índia } \\
\text { (Naxalbari) }\end{array}$ & Escolares & 331 & $3,63 \%$ & Manchas de Bitot & Bhattacharya et al., 2004 \\
\hline $\begin{array}{l}\text { Índia } \\
\text { (Nagpur - área urbana) }\end{array}$ & $<6$ anos & 308 & $35,7 \%$ & $I C C^{* *}$ & Khandait et al., 2000 \\
\hline $\begin{array}{l}\text { Índia } \\
\text { (Índios) }\end{array}$ & Pré-escolares & 1022 & $1,6 \%$ & M anchas de Bitot & Rao et al., 2005 \\
\hline Iraque & 0 a 6 anos & 700 & $29 \%$ & Xeroftalmia & Al-Kubaisy et al., 2002 \\
\hline $\begin{array}{l}\text { Sudeste da Ásia } \\
\text { ( } 8 \text { países) }\end{array}$ & Escolares & & $2,6 \%$ & Xeroftalmia branda & Singh \& West, Jr., 2004 \\
\hline \multicolumn{6}{|l|}{ África } \\
\hline \multirow[t]{2}{*}{ Mali } & Pré-escolares & 1510 & $4,3 \%$ & Cegueira noturna & Schémann et al., 2002 \\
\hline & & 1510 & $2 \%$ & M anchas de Bitot & \\
\hline \multirow[t]{5}{*}{ Etiópia } & Pré-escolares & 402 & $7,2 \%$ & Cegueira noturna, & Asrat et al., 2002 \\
\hline & e escolares & & $2,2 \%$ & M ancha de Bitot, & \\
\hline & & & $0,2 \%$ & Xerose corneal & \\
\hline & & & $0,5 \%$ & Ulceração corneal & \\
\hline & & & $0,5 \%$ & Cicatriz corneal & \\
\hline Etiópia (Norte) & 6 a 9 anos & 824 & $5,8 \%$ & Xeroftalmia & Kassaye et al., 2001 \\
\hline
\end{tabular}

*RS - Retinol Sérico; **ICC - Impressão Citológica Conjuntival. 


\section{Conclusões}

A prevalência deDVA foi registrada em crianças degrupos populacionais de vários estados brasileiros, principalmentenas regiões Sudestee N ordeste. Em algumas regiões de São Paulo, M inas Gerais e Sergipe, os limites de retinol sérico considerados baixos pela WHO alcançaram prevalências maiores do que $20 \%$ da população estudada, sendo que na Bahia atingiram mais de $40 \%$.

Não foram encontrados estudos relativos a sinais clínicos da DVA no Brasil, o que pode indicar inexistência de casos ou carência de pesquisas.

Nota-se que em algumas regiões do Brasil a DVA aparece como problema de saúde pública grave; entretanto, percebe-se prevalências mais elevadas em outros países.

Sugere-se que medidas de intervenção continuem sendo adotadas no Brasil como forma de prevenir e/ ou reduzir a DVA, tais como: educação nutricional eimplantação de programas desuple- mentação e fortificação de alimentos para populações de risco.

A nível mundial, os dados ainda são escassos, embora os estudos queabordam a DVA em crianças no mundo revelem que esta deficiência é um problema de saúde pública, principalmente nas regiões mais pobres do globo.

Asmaiores prevalências deDVA, segundo avaliação bioquímica, foram registradas em regiões da Etiópia, Nigéria e Ilhas M arshall chegando a $92,7 \%$ no Mali.

A suplementação com vitamina $A$ é uma me dida a curto prazo, efetiva para combater a DVA. Já a educação nutricional pode ser útil a longo prazo como complemento à suplementação e à fortificação dos alimentos.

Os diversos países devem se mobilizar para incrementar as ações que mantenham a DVA sob controle. A eliminação da DVA como problema desaúde pública éuma estratégia primordial para melhorar a sobrevivência, o crescimento e o de senvolvimento das crianças.

\section{Colaboradores}

RCRM M ilagres e LC Nunes trabalharam na concepção, pesquisa e redação do artigo. HM Pinheiro-Sant'Ana trabalhou na orientação e correção do artigo. 


\section{Referências}

1. World Health Organization (WHO). The Global Prevalence of Vitamin A Deficiency. Micronutrient Deficiency Information System (M DIS) Working Paper 2. Geneva: WHO; 1995.

2. World Health Organization (WHO). Nutrition for Health and Development: A global agenda for combating malnutrition. Progress Report. France: WH O; 2000.

3. Ramalho RA, Saunders C. Vitamina A: aspectos fisiopatológicos, diagnóstico e medidas de intervenção. Rev M etab Nutr 2003; 07:10-9.

4. Bloem MW, De Pee S, Danton-Hill ID. New issues in developing effective approaches for the prevention and control of vitamin A deficiency. Food Nut. Bull 1998; 19: 37-48.

5. Archana Dwivedi. Eliminating Vitamin A deficiency. UNICEF 2005 [acessado 2005 Set 10]. Disponível em: http://www.unicef.org/nutrition/23963 vitaminadeficiency.html

6. International Vitamin A Consultative Group (IVACG). Guidelines for the development of a simplified dietary assessment to identify groups at risk for inadequate intake for vitamin A. Washington, D.C.: Nutrition Foundation/ IVACG; 1989.

7. World Health Organization (WHO). Indicators for assessing vitamin A deficiency and their application in monitoring and evaluating intervention programmes. (M icronutrient Series,10). Geneva: WH O; 1996.

8. Ramalho RA, Flores H, Saunders C. Hipovitaminose A no Brasil: um problema de saúde pública. Rev Panam Salud Públ/ Pan Am J Public Health 2002; 12 (2):117-22.

9. Mclaren $D$, Frigg $M$. M anual de ver y vivir sobre los transtornos por deficiencia de vitamina A (VADD). Washington, D.C.: OPAS/OM S; 1999

10. Souza WA, Vilas Boas OM GC. A deficiência de vitamina A no Brasil: um panorama. Rev. Panam Salud Públ/ Pan Am J Public Health 2002; 12 (3):173-9.

11. Brasil. Ministério da Saúde. Secretaria de Políticas de Saúde. Política Nacional deAlimentação e Nutrição. Brasília: M inistério da Saúde; 2000.

12. Gonçalves-Carvalho CM R, Amaya-Farfan J, Wilke BC Vencovsky R. Prevalência dehipovitaminoseA em crianças da periferia do município de Campinas, São Paulo, Brasil. Cad Saúde Pública 1995; 11(1):85-96.

13. Souza QS, Sato K, Torres MAA. Detection of the prevalence of hipovitaminose $A$ in children under 2 years of age enrolled in basic health care units cities of state of São Paulo. Proceedings of the 16th Congress of Nutrition; 1998; M ontréal. p. 292.

14. Ferraz, IS; Daneluzzi, JC, Vannucchi H. Vitamin A deficiency in children aged 6 to 24 months in São Paulo state, Brazil. Nutr Res 2000; 20:757-68.

15. Sarni RS, Kochi C, Ramalho RA, Schoeps DO, Sato K, Mattoso LCQ et al. Vitamina A: nível sérico e ingestão dietética em crianças e adolescentes com déficit estatural de causa não hormonal. Rev Assoc M ed Bras 2002; 48(1):48-53.

16. Vítolo M R, Gama CM , Queiroz SS, Lopez FA, Colugnati FAB. Retinol sérico de adolescentes de uma escola da cidade de São Paulo. Rev Nutr 2004; 17(3):291-9.
17. Santos M A, Rezende EG, Lamounier JA, Galvão M AM , Bonomo $E$, Leite, RC. Hipovitaminose A em escolares da zona rural de M inas Gerais. Rev Nutr 2005; 18(3):331-9.

18. Ramalho RA, Anjos LA, Flores H. Hipovitaminose A em recém-nascidos em duas maternidades públicas no Rio de Janeiro, Brasil. Cad Saúde Pública 1998; $14(4): 821-7$

19. Ramalho RA, Anjos LA, Flores H. Valores séricos de vitamina $\mathrm{A}$ e teste terapêutico em préescolares atendidos em uma unidade de saúde do Rio de Janeiro, Brasil. Rev Nutr 2001; 14(1):5-12.

20. Ramalho RA, Saunders C, Natalizi DA, Cardoso LO, Accioly E. Níveis séricos de retinol em escolares de 7 a 17 anos no município do Rio de Janeiro. Rev Nutr 2004; 17(4):461-8.

21. Prado MS, Assis AM O, Cruz M M, Araújo N M P, Bonfim RIF, Pereira CME. Hipovitaminose A em crianças de áreas rurais do semi-árido baiano. Rev. Saúde Pública 1995; (4)29:295-300

22. Santos LM P, Assis AM O, M artins MC, Araújo M PN, M orris SS, Barreto M L. Situação nutricional e alimentar de préescolares no semi-árido da Bahia (Brasil): II Hipovitaminose A. Rev. Saúde Pública 1996; 30(1):67-74.

23. Assis AM O, Prado M S, Freitas M CS, M artins M C. Deficiência de vitamina A e desnutrição energético-protéica em crianças de localidades do semi-árido baiano. Rev Nutr 1997; 10:70-8.

24. Martins M C, Santos LM P, Assis, AM O. Prevalência da hipovitaminose A em préescolares no estado de Sergipe, 1998. Rev. Saúde Pública 2004; 38:537-42.

25. Diniz AS. Aspectos clínicos, subclínicos e epidemiológicos da hipovitaminose A no estado da Paraíba [tese de doutorado]. Recife (PE): Departamento de Nutrição, Centro de Ciências da Saúde da Universidade Federal de Pernambuco; 1997.

26. Instituto Nacional de Alimentação e Nutrição (INAN). Instituto Materno Infantil de Pernambuco (IMIP). II Pesquisa Estadual de Saúde e Nutrição: saúde, nutrição, alimentação e condições sócio-econômicas no estado de Pernambuco. Recife: INAN/IMIP; 1998.

27. Fernandes TFS, Diniz AS, Cabral PC, Oliveira RS, Lola M M F, Silva SM N et al. Hipovitaminose A em préescolares de creches públicas do Recife: indicadores bioquímico e dietético. Rev Nutr 2005; 18(4):471-80.

28. Alencar FH, Castro JS, Yuyama LKO, Marinho HA, Nagahama $\mathrm{D}$. Diagnóstico da realidade nutricional no estado do Amazonas, Brasil. I - Hipovitaminose A. Acta Amaz 2002; 32:613-23.

29. Portaria no 2.160 de 29 de Dezembro de 1994. Cria, no Instituto Nacional de Alimentação e Nutrição (INAN), o Programa Nacional de Controle das Deficiências de Vitamina $\mathrm{A}$ e dá outras providências. Diário $\mathrm{O}$ ficial da União 1994; 30 dez.

30. Portaria $n^{\circ} 729$ de 13 de Maio de 2005. Institui o Programa Nacional de Suplementação de Vitamina A e dá outras providências. Diário Oficial da União, 16 maio de 2005. 
31. World Health Organization (WHO). Integration of vitamin A supplementation with immunization: policy and programmeimplications. Report of a meeting. N ew York: WHO; 1998.

32. Radhika M S, Bhaskaram P, Balakrishna N, Ramalakshmi BA, Devi S, Kumar BS. Effects of vitamina A deficiency during pregnancy on maternal and child health. BJOG 2002; 109: 689-93.

33. West Jr KP. Extent of vitamin A Deficiency among preschool children and women of reproductiveage.J Nutr 2002; 132:2857-66.

34. Ramakrishnan U. Prevalence of micronutrient malnutrition worldwide. Nutr Rev 2002; 60: 46-52.

35. Azaïs-Braesco V, Pascal G. Vitamin A in pregnancy: requirements and safety limits. Am J Clin Nutr 2000; 71(Supl 5):1325-33.

36. Thurnham DI, Mc Cabe GP, Northrop-Clewes, Nestel $P$. Effects of subclinical infection on plasma retinol concentrations and assessment of prevalence of vitamin A deficiency: meta-analysis. Lancet 2003; 362:2052-8.

37. Singh V, West Jr KP. Vitamin A deficiency and xerophthalmia among school-aged children in Southeastern Asia. Eur J Clin Nutr 2004; 58:1342-9.

38. Mora JO, Gueri M, M ora OL. Vitamin A deficiency in Latin America and the Caribbean: An overview. Rev Panam Salud Públ/ Pan AmJ Public H ealth 1998; 4:176186.

39. De Navarro LC, Nicholls S. D eficiência de hierro, vitamina A y prevalencia de parasitismo intestinal em la poblacion infantil de Colômbia. Informe de Republica de Colombia, M inesterio de Salud, Instituto Nacional de Salud, Subdirección de Investigación y Desarrollo, Laboratorio de Nutrición. Bogota: Minesterio de Salud; 1996.

40. Villalpando S, M ontalvo-Velardel,Zambrano N, GarcíaGuerra A, Ramírez-Silva Cl, Shamah-Levy T et al. Vitamins $A$, and $C$ and folate status in Mexican children under 12 years and women $12-49$ years: A probabilistic national survey. Salud Publica M ex 2003; 45 (Supl 4):50818.

41. Paez Valery M C, Solano L, Del Real S. Risk indicators of vitamin $A$ deficiency in children younger than 15 -years old from a slum area of Valencia, Venezuela. Arch Latinoam Nutr 2002; 52:12-9.

42. Castejon HV, Ortega P, Amava D, Gomez G, Leal J, Caste jon OJ. Co-existence of anemia, vitamin $A$ deficiency and growth retardation among children 24-84 months old in Maracaibo, Venezuela. Nutr Neurosci 2004; 7:113-9.

43. Castejon HV, Ortega P, Diaz M E, Amava D, Gomez G, Ramos $M$ et al. Prevalence of sub-clinical vitamin $A$ deficiency and malnutrition in slum children in $\mathrm{M}$ aracaibo - Venezuela. Arch Latioam Nutr 2001; 51:25-32.

44. Stephens D, Jackson PL, Gutierrez Y. Subclinical vitamin A deficiency. A potentially unrecognized problem in the United States. Pediatr Nurs 1996; 22:377-456.

45. Stephenson LS, Latham MC \& Ottesene EA. Global malnutrition. Parasitology 2000; 121 (Suppl):5-22.
46. Feungpean B, Suthutvoravut U, Supapannachart S, Rakthai S, Chatvuttinum S. Vitamin A status in premature infants. J M ed Assoc Thai 2002; 85(Supl 4):1219-23.

47. Coles CL, Levy A, Gorodischer R, Dagan R, Deckelbaum RJ, Blaner WS et al. Subclinical vitamin A deficiency in Israeli-Bedouin toddlers. Eur J Clin Nutr 2004; 58:796-802.

48. Rao VG, Yadav R, Dolla CK, Kumar S, Bhondeley MK Ukey M. Undernutrition \& childhood morbidities among tribal preschool children. Indian J M ed Res 2005; 122:43-7.

49. Toteja GS, Singh P, Dhillon BS, Saxena BN. Vitamin A deficiency disorders in 16 districts of India. Indian J Pediatr 2002; 69:603-5.

50. Bhattacharya RN, Shrivastava P, Sadhukhan SK, Lahiri SK, Chakravorty M, Saha JBPC. Sen M emorial best paper award on rural health practice: A study on visual acuity and vitamin A deficiency among primary school students in Naxalbari Village, darjeeling district of West Bengal. Indian J Public Health 2004; 48:171-80.

51. Khandait DW, Vasudeo ND, Zodpey SP, Kumbhalkar DT. Risk factors for subclinical vitamin A deficiency in children under the age of 6 years. J Trop Pediatr 2000; 46:239-41.

52. Al-Kubaisy W, Al-Rubaiy M G, Nassief HA. Xerophthalmia among hospitalized Iraqi children. East M editerr Health I 2002; 8:496-502.

53. Tan Z, M a G, Lin L, Liu C, Liu Y, JiangJ et al. Prevalence of subclinical vitamin A deficiency and its affecting factors in 8669 children of China. Zhonghua Yu Fang Y Xue Za Zhi 2002; 36:161-3.

54. Khatib IM. High prevalence of subclinical vitamin A deficiency in Jordan: a forgotten risk. Food Nutr Bull 2002; 23 (Supl 3):228-36.

55. Khan M A, Khan M D. Classification of 154 clinical cases of vitamin A deficiency in children (0-15 years) in a tertiary hospital in North West Frontier Province Pakistan. J Pak M ed Assoc 2005; 55:77-8.

56. Mihora LD, Jatla KK, Little T, Campbell M, Rahim A, Enzenauer RW. Vitamina A deficiency in Afghanistan. Eye Contact Lens 2004; 30:159-62.

57. Pedro M R, M adriaga JR, Barba CV, Habito RC, Gana $A E$, Deitcher $M$ et al. The national Vitamin A Supplementation Program and subclinical vitamin A deficiency among preschool children in the Philippines. Food N utr Bull 2004; 25:319-29.

58. Khan NC, Khoi HH, Giav T, N han NT, N han NT, Dung $\mathrm{NC}$ et al. Control of vitamin A deficiency in Vietnam: achievements and future orientation. Food Nutr Bull 2002; 23:133-42.

59. Farbos S, Resnikoff S, Peyramaure F, Castan R. Xe rophthalmia. Identification des populations à risque intermédiaire. Cahiers Santé 1995; 5:159-61.

60. Schémann JF, Banou AA, Guindo A, Joret V, Traore L, Malvy $D$. Prevalence of Undernutrition and vitamin $A$ deficiency in the Dogon Region, M ali. American College of Nutrition 2002; 21:381-7. 
61. Oso OO, Abiodun PO, Omotade OO Oyewole D. Vitamin A status and Nutritional Intake of carotenoids of Preschool Children in Ijaye Orile Community in Nige ria. J Trop Pediatr 2003; 49:42-7.

62. Rabiu MM, Kyari F. Vitamin A deficiency in Nigeria. Niger J Med 2002; 11:6-8.

63. Aguayo VM , Kahn S, Ismael C, M eershoek S. Vitamin A deficiency and child mortality in Mozambique. Public Health Nutr 2005; 8:29-31.

64. Kassaye T, Receveur O, Johns T, Becklake M R. Prevalence of vitamin A deficiency in children aged 6-9 years in Wukro, Northern Ethiopia. Bull World Health Organ 2001; 79: 415-22.

65. Asrat YT, Omwega AM, Muita JW. Prevalence of vitamin A deficiency among pre-school and school-aged children in Arssi Zone, Ethiopia. East Afr M ed J 2002; 79:501.
66. Kello AB, Gilbert $C$. causes of severe visual impairment and blindnessin children in schools for theblind in Ethiopia. Br J O phthalmol 2003; 87:526-30.

67. GregoryJR, Collins DL, Daves PSW, Hughes JM, Clarke PC. National diet and Nutrition survey: children aged 1 1/2 to 4 1/2 years. London: H M SO, 1995.

68. Palafox NA, Gamble MV, Dancheck B, Ricks M O, Briand K, Semba RD. Vitamin A Deficiency, Iron Deficiency, and Anemia Among Preschool Children in the Republic of the M arshall Islands. Nutrition 2003; 19:405- 8.

69. International Vitamin A Consultative Group (IVACG). Vitamin $A$ and the common agenda for micronutrients. Lima, Peru: IVACG; 2004.

70. United Nations Children's Fund (UNICEF). Ending vitamin A deficiency: A challenge to the world 2001 [acessado 2006 Ago 02]. Disponível em: http://www. unicef.org/publications/files/Vitamin_A_Goals. pdf

Artigo apresentado em 11/04/2006

Aprovado em 13/07/2006

Versão final apresentada em 12/09/2006 